\title{
Study of dilepton production in association with leading hadron at RHIC and LHC energies
}

\author{
Eduardo Basso \\ Instituto de Física, Universidade Federal do Rio de Janeiro, Caixa Postal 68528, Rio de Janeiro, \\ RJ 21941-972, Brazil \\ Department of Astronomy and Theoretical Physics, Lund University, SE-223 62 Lund, Sweden \\ E-mail: eduardo.basso@thep.lu.se
}

\section{Victor P. Goncalves}

Department of Astronomy and Theoretical Physics, Lund University, SE-223 62 Lund, Sweden High and Medium Energy Group, Instituto de Física e Matemática, Universidade Federal de Pelotas, Pelotas, RS, 96010-900, Brazil

E-mail: victorpbgethep.lu.se

\section{Jan Nemchik}

Czech Technical University in Prague, FNSPE, Břehová 7, 11519 Prague, Czech Republic Institute of Experimental Physics SAS, Watsonova 47, 04001 Košice, Slovakia

E-mail: nemcikesaske.sk

\section{Roman Pasechnik}

Department of Astronomy and Theoretical Physics, Lund University, SE-223 62 Lund, Sweden

E-mail: roman.pasechnik@thep.lu.se

\section{Michal Šumbera *}

Nuclear Physics Institute ASCR, 25068 Řež, Czech Republic

E-mail: sumbera@ujf.cas.cz

We demonstrate that current experimental data on $\gamma / Z^{0}$ and heavy quarkonium production in $p p$ collisions at RHIC and LHC energies can be described by the color dipole formalism. Three different phenomenological models based on saturation physics are used to estimate the DrellYan (DY) dilepton spectra in such processes. In order to further constrain QCD dynamics we suggest to measure the azimuthal correlation function $C(\Delta \phi)$ between mid-rapidity DY pairs and central/forward pions. On the other hand, this is a key background for $C$-odd heavy quarkonia (e.g. $J / \psi$ and $\Upsilon$ ) production at high $p_{T}$ 's which can be accompanied by a high- $p_{T}$ hadron from fragmentation of an additional gluon according to the Color-Singlet model (CSM). As a first step, we have estimated the transverse momentum distributions $J / \psi$ and $\Upsilon$ in the dipole CSM and found a reasonable agreement with data at various energies. Similarly to the DY case we suggest to study correlations between a high- $p_{T}$ jet and $J / \psi$ or $\Upsilon$ in the dilepton channel at mid-rapidities.

The European Physical Society Conference on High Energy Physics

22-29 July 2015

Vienna, Austria

\footnotetext{
* Speaker.
} 
Study of dynamics of colored partons at small/medium momentum transfers is one of the important frontiers of Quantum Chromo Dynamics (QCD) today. The DY pair production process in $p p / p A / A A$ collisions is a prominent tool in studies of strong interaction dynamics in an extended kinematical range of energies and rapidities (for a recent review see, e.g. Ref. [1]). In particular, such a process directly probes the parton distribution functions (PDFs) as well as soft QCD dynamics and non-linear effects.

On the other hand, the DY process is a background process in studies of production of such heavy quarkonia as $J / \psi$ and $\Upsilon$ in the dilepton decay channel. The latter serves as an efficient probe for non-perturbative properties of the color medium. A detailed study of production mechanisms of heavy quark pair in different color and parity states resulting in $\chi_{c}, J / \psi, \psi^{\prime}$, etc. is of particular interest and has been discussed in many articles so far (see e.g. Refs. [2, 3]). Despite a large variety of different approaches, a universality between corresponding descriptions remains questionable, especially, when it concerns an underlying QCD production mechanism for $C$-odd states such as $J / \psi, \psi^{\prime}$ and $\Upsilon$.

Since both types of the processes, virtual gauge $\gamma^{*} / Z^{0}$ boson and heavy quarkonia production, are typically studied in the DY channel, we propose to investigate their common signatures, in particular, the $p_{T}$ spectra and azimuthal dilepton-leading hadron correlations on the same footing. For this purpose, we employ the phenomenological color dipole approach which effectively takes into account the major part of the higher-order QCD corrections [4]. At high energies, color dipoles with a definite transverse separation are eigenstates of interaction. The main ingredient of the dipole formalism is the process-independent universal dipole-target scattering cross section. It can thus be determined phenomenologically, for example, from the Deep Inelastic Scattering (DIS) data [5]. The dipole approach is formulated in the target rest frame where the DY process can be viewed as a bremsstrahlung of a virtual $\gamma^{*} / Z^{0}$ boson off a projectile quark line [6, 7]. In the high-energy limit, the projectile quark looses a small fraction of its momentum and can further fragment into a forward leading hadron, e.g. a pion, which can then be efficiently detected at RHIC Run II experiment in the $\pi^{0} \rightarrow 2 \gamma$ channel. Such an important observable as the correlation function in azimuthal angle between the forward pion and the mid-rapidity dilepton is sensitive to the saturation effects and thus probes the properties of the dense gluonic field in the target $[8,9,10]$.

The differential cross section for inclusive $\gamma, Z^{0}$ production with invariant mass $M$ and transverse momentum $p_{T}$ is expressed in terms of the projectile quark (antiquark) densities $q_{f}\left(\bar{q}_{f}\right)$

$$
\frac{d \sigma\left(p p \rightarrow G^{*} X\right)}{d^{2} p_{T} d x_{F}}=\frac{x_{1}}{x_{1}+x_{2}} \sum_{f, \bar{f}} \sum_{\lambda_{G}=L, T} \int_{x_{1}}^{1} \frac{d \alpha}{\alpha^{2}} q_{f}\left(x_{1} / \alpha, \mu_{F}^{2}\right) \frac{d \sigma_{\lambda_{G}}^{f}\left(q N \rightarrow q G^{*} X\right)}{d \ln \alpha d^{2} p_{T}}
$$

where $x_{F}=x_{1}-x_{2}$ is the Feynman variable, $\alpha$ and $x_{1}$ are the light-cone momentum fractions carried by the gauge $G=\gamma, Z^{0}$ boson off the projectile (anti)quark and proton, respectively, $\mu_{F}^{2}=$ $p_{T}^{2}+\left(1-x_{1}\right) M^{2} \simeq M^{2}$ is the factorization scale in quark PDFs. The dilepton cross section is then related to the inclusive $G=\gamma, Z^{0}$ production cross section (1) as follows

$$
\frac{d \sigma\left(p p \rightarrow\left[G^{*} \rightarrow l \bar{l}\right] X\right)}{d^{2} p_{T} d M^{2} d x_{F}}=\mathscr{F}_{G}(M) \frac{d \sigma\left(p p \rightarrow G^{*} X\right)}{d^{2} p_{T} d x_{F}},
$$

where

$$
\mathscr{F}_{\gamma}(M)=\frac{\alpha_{e m}}{3 \pi M^{2}}, \quad \mathscr{F}_{Z}(M)=\operatorname{Br}\left(Z^{0} \rightarrow l \bar{l}\right) \rho_{Z}(M)
$$


Here, the branching ratio $\operatorname{Br}\left(Z^{0} \rightarrow l \bar{l}\right) \simeq 0.101$, and $\rho_{Z}(M)$ is the invariant mass distribution of the $Z^{0}$ boson in the narrow width approximation

$$
\rho_{Z}(M)=\frac{1}{\pi} \frac{M \Gamma_{Z}(M)}{\left(M^{2}-m_{Z}^{2}\right)^{2}+\left[M \Gamma_{Z}(M)\right]^{2}}, \quad \Gamma_{Z}(M) / M \ll 1,
$$

in terms of the on-shell $Z^{0}$ boson mass, $m_{Z} \simeq 91.2 \mathrm{GeV}$, and the generalized total $Z^{0}$ decay width

$$
\Gamma_{Z}(M)=\frac{\alpha_{e m} M}{6 \sin ^{2} 2 \theta_{W}}\left(\frac{160}{3} \sin ^{4} \theta_{W}-40 \sin ^{2} \theta_{W}+21\right),
$$

where $\theta_{W}$ is the Weinberg gauge boson mixing angle in the SM, $\sin ^{2} \theta_{W} \simeq 0.23$, and $\alpha_{e m}=$ $e^{2} /(4 \pi)=1 / 137$ is the fine structure constant.

In the framework of the dipole approach, the gauge boson transverse momentum distribution can be obtained by a straightforward generalization of the well-known formulas for the photon bremsstrahlung $[7,11]$. For a given incoming quark flavour $f$ it reads

$$
\begin{aligned}
\frac{d \sigma_{T, L}^{f}\left(q N \rightarrow q G^{*} X\right)}{d \ln \alpha d^{2} p_{T}} & =\frac{1}{(2 \pi)^{2}} \sum_{\text {quark pol. }} \int d^{2} \rho_{1} d^{2} \rho_{2} e^{i \mathbf{p}_{T} \cdot\left(\boldsymbol{\rho}_{1}-\boldsymbol{\rho}_{2}\right)} \Psi_{T, L}^{V-A}\left(\alpha, \boldsymbol{\rho}_{1}, m_{f}\right) \Psi_{T, L}^{V-A, *}\left(\alpha, \boldsymbol{\rho}_{2}, m_{f}\right) \\
& \times \frac{1}{2}\left[\sigma_{q \bar{q}}\left(\alpha \boldsymbol{\rho}_{1}, x_{2}\right)+\sigma_{q \bar{q}}\left(\alpha \boldsymbol{\rho}_{2}, x_{2}\right)-\sigma_{q \bar{q}}\left(\alpha\left|\boldsymbol{\rho}_{1}-\boldsymbol{\rho}_{2}\right|, x_{2}\right)\right]
\end{aligned}
$$

where $\Psi_{T, L}^{V, A}$ are the vector $(\mathrm{V})$ and axial (A) distribution amplitudes for the bremsstrahlung $q \rightarrow$ $q+G$ with transversely (T) and longitudinally (L) polarised gauge boson (found e.g. in Ref. [10]), respectively, $\boldsymbol{\rho}_{1}$ and $\boldsymbol{\rho}_{2}$ are the quark- $G$ transverse separations in the total radiation amplitude and its conjugated counterpart, respectively, $\sigma_{q \bar{q}}\left(r, x_{2}\right)$ is the universal dipole cross section known from phenomenology.

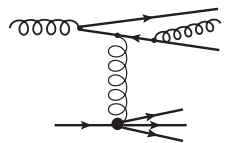

(1)

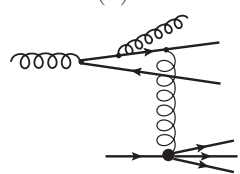

(4)

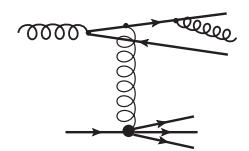

(2)

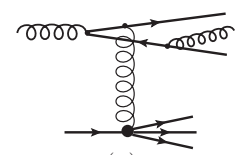

(5)

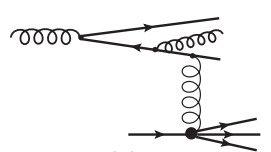

(3)

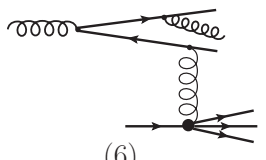

(6)

Figure 1: The leading-order contributions to the $C$-odd $S$-wave (e.g. $J / \psi, \psi^{\prime}$ and $\Upsilon$ ) quarkonia production.

Moving now to the heavy quarkonia production let us note that replacement of virtual photon with a gluon allows us to describe it as a process when the projectile hadron develops a fluctuation which contains a heavy quark pair $Q \bar{Q}$. Interaction with the color field of the target proton (nucleus) then may release these heavy quarks [12]. Leading order contribution to $C$-odd $S$-wave quarkonium production is shown on Fig. 1. The extra gluon emission off the produced heavy quark $Q \bar{Q}$ pair which is needed to prepare $\{Q \bar{Q}\}_{1+}$ state goes beyond simple Born approximation valid for $P$-wave quarkonia and open charm production. In analogy to the gauge boson production cross section (6), the corresponding Born amplitudes for the $G \rightarrow\{Q \bar{Q}\}_{1^{+}}+G$ fluctuation (where $Q \bar{Q}$ is in the $C$-odd 
state) can be straightforwardly generalised to the dipole formula (dipole CSM). For that purpose, it is sufficient to assume that the radiated gluon fraction $\alpha_{3}$ is small compared to the charm quark fraction $\alpha$ in the quarkonium wave function, i.e. $\alpha_{3} \ll \alpha$, as was done in Ref. [13].
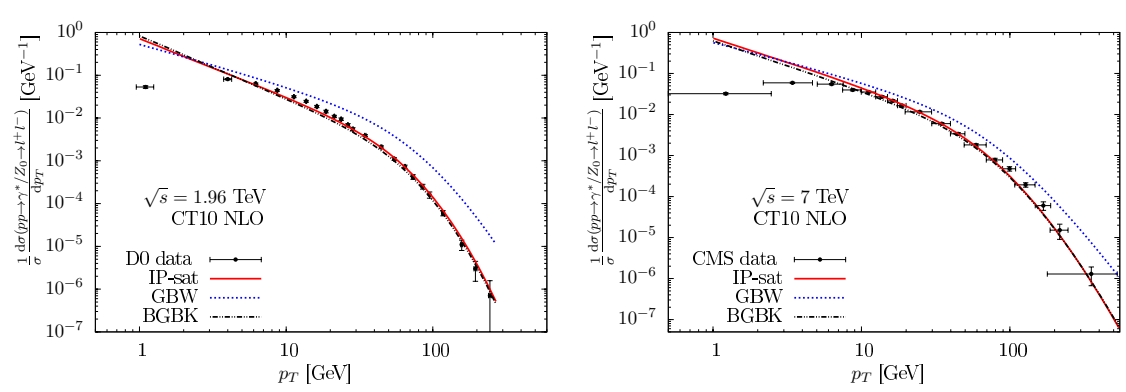

Figure 2: The transverse momentum distributions of $Z^{0}$ bosons in $p p$ collisions at $\sqrt{s}=1.96 \mathrm{TeV}$ (left panel) and $7 \mathrm{TeV}$ (right panel) versus data from the D0 [14] and CMS [15] Collaborations. The CT10 PDFs [16] were used.

In what follows, we consider two distinct phenomenological models taking into account the DGLAP evolution of the gluon PDF in the target as well as the saturation effects. The BGBK dipole cross section proposed in Ref. [17] reads

$$
\sigma_{q \bar{q}}(\rho, x)=\sigma_{0}\left[1-\exp \left(-\frac{\pi^{2}}{\sigma_{0} N_{c}} \rho^{2} \alpha_{s}\left(\mu^{2}\right) x g\left(x, \mu^{2}\right)\right)\right],
$$

where $N_{c}=3$ is the number of colors, $\alpha_{s}\left(\mu^{2}\right)$ and $x g\left(x, \mu^{2}\right)$ are the strong coupling constant and the gluon PDF, respectively, determined at the scale $\mu^{2}$ which is related to the dipole size $\rho$ as $\mu^{2}=C / \rho^{2}+\mu_{0}^{2}$, with $C, \mu_{0}$ and $\sigma_{0}$ parameters fitted to HERA data. The gluon density at initial scale $\mu_{0}^{2}$ is parametrized as

$$
x g\left(x, \mu_{0}^{2}\right)=A_{g} x^{-\lambda_{g}}(1-x)^{5.6} .
$$

The best fit values of the model parameters are the following: $A_{g}=1.2, \lambda_{g}=0.28, \mu_{0}^{2}=0.52$ $\mathrm{GeV}^{2}, C=0.26$ and $\sigma_{0}=23 \mathrm{mb}$. The second version called IP-SAT is a generalization of the BGBK cross section accounting for the impact parameter dependence in Ref. [18]. Finally, the DY cross sections results of the BGBK and IP-SAT models are compared to the corresponding GBW predictions [5].

Let us turn to a discussion of numerical results. In Fig. 2 we present our predictions for the dilepton $p_{T}$ distribution of $Z^{0}$ bosons in $p p / p \bar{p}$ collisions at Tevatron $\sqrt{s}=1.96 \mathrm{TeV}$ (left panel) and $7 \mathrm{TeV}$ (right panel). At large $p_{T}>5 \mathrm{GeV}$ the data are well described by the DGLAP-evolved dipole models IP-SAT and BGBK, but not GBW. At low $p T$ 's the data are not described which may indicate an importance of the Sudakov resummation and primordial transverse momentum effects not accounted for in this calculation.

Now let us switch to investigation of the correlation function in $p p$ collisions. Fig. 3 shows our predictions for the azimuthal dilepton-pion correlation function $C(\Delta \phi)$ in the range of low invariant masses dominated by the virtual photon channel, $\gamma^{*} \rightarrow l \bar{l}$. The figure demonstrates that the double peak structure emerges in $p p$ collisions at RHIC and LHC energies considering different values of the photon (central) and pion (forward) rapidities. The double-peak structure of $C(\Delta \phi)$ arises 

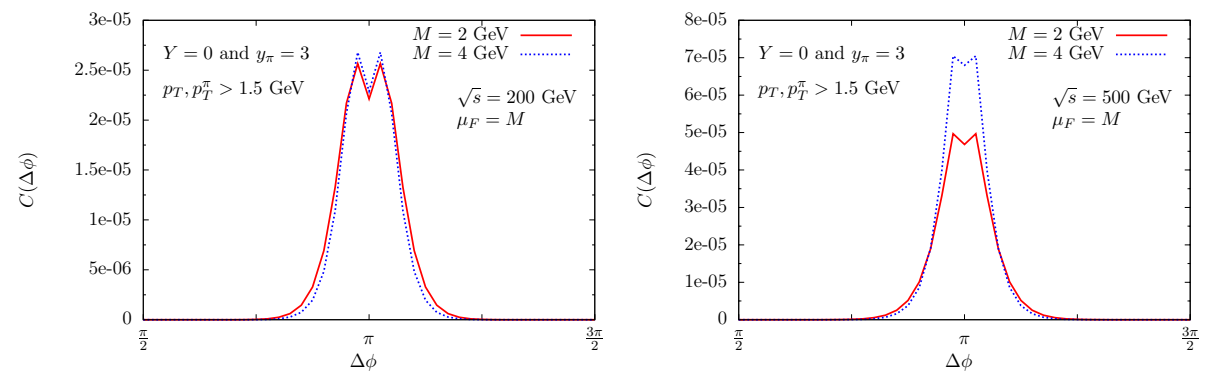

Figure 3: The correlation function $C(\Delta \phi)$ for the the associated DY pair and pion production in $p p$ collisions at RHIC $(\sqrt{s}=200,500 \mathrm{GeV})$ for different values of the photon and pion rapidities. The GBW model is used here. Constraints on dilepton $p_{T}$ and rapidity $Y$ and on pion $p_{T}^{\pi}$ and rapidity $y_{\pi}$ are indicated.
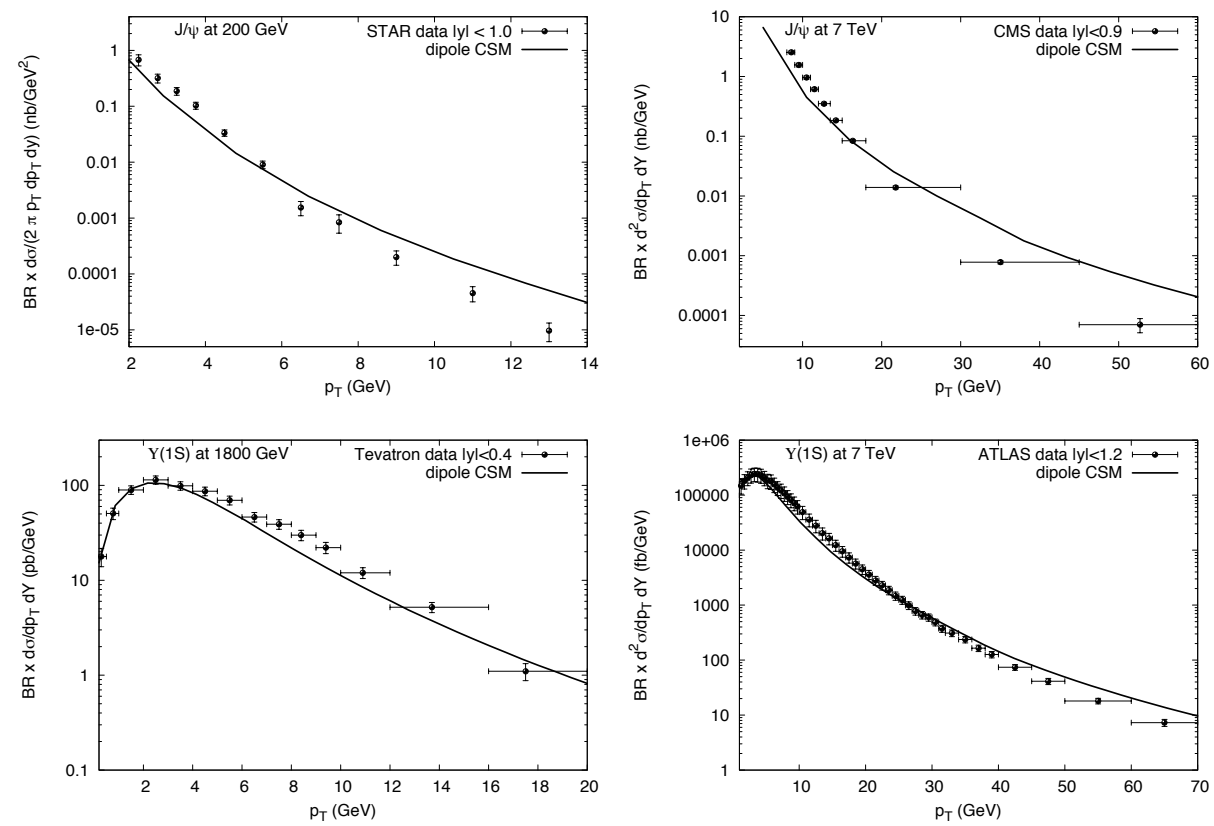

Figure 4: The transverse momentum distributions of the $J / \psi \rightarrow \mu^{+} \mu^{-}$(top row) and $\Upsilon \rightarrow \mu^{+} \mu^{-}$(bottom row) versus data from the STAR [19] and CMS [20] collaborations for prompt $J / \psi$ uproduction and from Tevatron [21] and ATLAS [22] collaborations for $\Upsilon$ production, respectively. The GBW model is used here.

only for pions at large forward rapidities, where the saturation scale takes values of the order of the dilepton invariant mass. At the same time, the width of a double peak arround $\Delta \phi \simeq \pi$ is strongly correlated with the magnitude of the saturation scale $Q_{s}$. It is important to emphasize that such forward-central correlations can be experimentally studied by the STAR Collaboration in both $p p$ and $p A$ collisions.

Finally, in Fig. 4 the preliminary results for the transverse momentum distributions of $J / \psi$ (upper row) and $\Upsilon$ (lower row) in association with an unobserved (gluonic) jet in the dipole CSM versus available data are shown for various energies. While the predicted shapes appeared to be somewhat flatter than the data, an overall normalisation for both types of $C$-odd quarkonia and 
its energy dependence are well reproduced. A further more detailed analysis of the primordial transverse momentum effects and other theory uncertainties is necessary.

\section{Acknowledgements}

E. B. is supported by CAPES and CNPq (Brazil), contract numbers 2362/13-9 and 150674/20155. V. P. G. has been supported by CNPq, CAPES and FAPERGS, Brazil. R. P. is supported by the Swedish Research Council, contract number 621-2013-428. J. N. is partially supported by the grant 13-20841S of the Czech Science Foundation (GAČR), by the Grant MSMT LG13031, by the Slovak Research and Development Agency APVV-0050-11 and by the Slovak Funding Agency, Grant 2/0020/14. M. Š. is supported by the grant LG 13031 of the Ministry of Education of the Czech Republic and by the grant 13-20841S of the Czech Science Foundation (GACR).

\section{References}

[1] J. C. Peng and J. W. Qiu, Prog. Part. Nucl. Phys. 76, 43 (2014)

[2] M. Butenschoen and B. A. Kniehl, Phys. Rev. Lett. 107, 232001 (2011)

[3] Y. Feng, J. P. Lansberg and J. X. Wang, Eur. Phys. J. C 75, no. 7, 313 (2015)

[4] N. N. Nikolaev, B. G. Zakharov, Z. Phys. C 64, 631 (1994).

[5] K. J. Golec-Biernat, M. Wusthoff, Phys. Rev. D 59, 014017 (1998).

[6] S. J. Brodsky, A. Hebecker and E. Quack, Phys. Rev. D 55, 2584 (1997)

[7] J. Raufeisen, J. -C. Peng and G. C. Nayak, Phys. Rev. D 66, 034024 (2002).

[8] A. Stasto, B-W Xiao and D. Zaslavsky, Phys. Rev.D 86, 014009 (2012).

[9] J. Jalilian-Marian and A. H. Rezaeian, Phys. Rev. D 86, 034016 (2012); A. H. Rezaeian, Phys. Rev. D 86, 094016 (2012).

[10] E. Basso, V. P. Goncalves, J. Nemchik, R. Pasechnik and M. Sumbera, arXiv:1510.00650 [hep-ph].

[11] B. Z. Kopeliovich, J. Raufeisen, A. V. Tarasov and M. B. Johnson, Phys. Rev. C 67, 014903 (2003).

[12] J. Raufeisen, J. C. Peng Phys. Rev. D 67, 054008 (2003)

[13] B. Kopeliovich, A. Tarasov and J. Hufner, Nucl. Phys. A 696, 669 (2001).

[14] V. Abazov et al. (D0 Collaboration), Phys. Rev. Lett. 100, 102002 (2008).

[15] S. Chatrchyan et al. [CMS Collaboration], Phys. Rev. D 85, 032002 (2012).

[16] H. L. Lai et al, Phys. Rev. D 82, 074024 (2010).

[17] J. Bartels, K. Golec-Biernat, and H. Kowalski, Phys. Rev. D 66, 014001 (2002).

[18] H. Kowalski, L. Motyka and G. Watt, Phys. Rev. D 74, 074016 (2006); G. Watt and H. Kowalski, Phys. Rev. D 78, 014016 (2008).

[19] L. Adamczyk et al. [STAR Collaboration], Phys. Lett. B 722, 55 (2013).

[20] S. Chatrchyan et al. [CMS Collaboration], JHEP 1202, 011 (2012).

[21] D. Acosta et al. [CDF Collaboration], Phys. Rev. Lett. 88, 161802 (2002).

[22] G. Aad et al. [ATLAS Collaboration], Phys. Rev. D 87, no. 5, 052004 (2013). 